BMJ Open

Diabetes

Research

\& Care

\title{
Causal associations of insulin resistance with coronary artery disease and ischemic stroke: a Mendelian randomization analysis
}

\author{
Weiqi Chen, ${ }^{1,2}$ Shukun Wang, ${ }^{3}$ Wei Lv, ${ }^{2}$ Yuesong Pan (1) ${ }^{1,2}$
}

To cite: Chen W, Wang S, Lv W, et al. Causal associations of insulin resistance with coronary artery disease and ischemic stroke: a Mendelian randomization analysis. BMJ Open Diab Res Care 2020;8:e001217. doi:10.1136/ bmjdrc-2020-001217

- Additional material is published online only. To view, please visit the journal online (http://dx.doi.org/10.1136/ bmjdrc-2020-001217).

WC and SW contributed equally.

Received 22 January 2020 Revised 2 April 2020 Accepted 26 April 2020

\section{Check for updates}

C Author(s) (or their employer(s)) 2020. Re-use permitted under CC BY-NC. No commercial re-use. See rights and permissions. Published by BMJ.

${ }^{1}$ Department of Neurology, Beijing Tiantan Hospital, Capital Medical University, Beijing, China

${ }^{2}$ China National Clinical Research Center for Neurological Diseases, Beijing Tiantan Hospital, Capital Medical University, Beijing, China

${ }^{3}$ The Brain Science Center, Beijing Institute of Basic Medical Sciences, Beijing, China

Correspondence to Dr Yuesong Pan; yuesongpan@ncrcnd.org.cn

\section{ABSTRACT}

Introduction The relationship between insulin resistance (IR) and cardiovascular diseases is unclear. We aimed to examine the causal associations of IR with cardiovascular diseases, including coronary artery disease, myocardial infarction, ischemic stroke and its subtypes, using Mendelian randomization.

Research design and methods Due to low sample size for gold standard measures and in order to well reflect the underlying phenotype of $\mathrm{IR}$, we used 53 single nucleotide polymorphisms associated with IR phenotypes (ie, fasting insulin, high-density lipoprotein cholesterol and triglycerides) from recent genome-wide association studies (GWASs) as instrumental variables. Summary-level data from four GWASs of European individuals were used. Data on IR phenotypes were obtained from meta-analysis of GWASs of up to 188577 individuals and data on the outcomes from GWASs of up to 446696 individuals. Mendelian randomization (MR) estimates were calculated with inverse-variance weighted, simple and weightedmedian approaches and MR-Egger regression was used to explore pleiotropy.

Results Genetically predicted 1-SD increase in IR phenotypes were associated with a substantial increase in risk of coronary artery disease $(0 \mathrm{R}=1.79,95 \% \mathrm{Cl}$ : 1.57 to $2.04, p<0.001)$, myocardial infarction $(0 R=1.78$, $95 \% \mathrm{Cl}: 1.54$ to $2.06, \mathrm{p}<0.001)$, ischemic stroke $(\mathrm{OR}=1.21$, $95 \% \mathrm{Cl}: 1.05$ to $1.40, \mathrm{p}=0.007$ ) and the small-artery occlusion subtype of stroke $(\mathrm{OR}=1.80,95 \% \mathrm{Cl}: 1.30$ to $2.49, p<0.001$ ), but not associated with the large-artery atherosclerosis and cardioembolism subtypes of stroke. There was no evidence of pleiotropy. Results were broadly consistent in sensitivity analyses using simple and weighted-median approaches accounting for potential genetic pleiotropy.

Conclusions This study provides evidence to support that IR was causally associated with risk of coronary artery disease, myocardial infarction, ischemic stroke and the small-artery occlusion subtype of stroke.

\section{INTRODUCTION}

Insulin resistance (IR) is the clinical state of a reduced sensitivity to insulin with an impaired ability of insulin to maintain normal glucose metabolism. IR is a complex trait, whereas high fasting insulin levels, low high-density

\section{Significance of this study}

What is already known about this subject?

- Previous observational studies showed that insulin resistance was associated with an increased risk of cardiovascular diseases.

- Whether this reflects a causal association remains to be established.

What are the new findings?

- Genetically predicted insulin resistance phenotypes was associated with an increased risk of coronary artery diseases, myocardial infarction, ischemic stroke and the small-artery occlusion subtype of stroke.

How might these results change the focus of research or clinical practice?

- Mechanism underlying the association of insulin resistance with cardiovascular diseases requires further investigation.

- Further validations are needed in studies with large sample sizes for the risk of stroke subtypes.

lipoprotein cholesterol (HDL-C) levels and high triglycerides (TGs) levels are three hallmarks of common IR. ${ }^{12}$ Due to absence of well-powered genome-wide association studies (GWASs) for gold standard measures of IR derived from euglycemic clamp and in order to well reflect the underlying phenotypes of IR, Lotta $e t a l^{2}$ identified 53 genetic variants for IR based on these three phenotypes, and Wang et a $\hat{\imath}$ further generated a composite genetic instrument for IR phenotypes by meta-analyzing these genetic variants.

IR is considered as a key risk factor of adverse metabolic and cardiovascular disease. ${ }^{4} 5$ Previous observational studies showed that IR was positively associated with an increased risk of coronary artery diseases $(\mathrm{CAD})^{6-8}$ and ischemic stroke $\mathrm{e}^{910}$ in the general population. However, positive association was not observed in other studies. ${ }^{11}$ Whether this reflects a 
causal association remains to be established since observational epidemiological studies suffer from potential biases and reverse causation which limits their ability to robustly identify causal associations. ${ }^{12}$ Whereas, recent clinical trials also demonstrated that insulin sensitizing agents that ameliorated IR prevented vascular events. ${ }^{13} 14$ Previous Mendelian randomization (MR) analyses also showed causal associations of IR-related traits (diabetes and obesity) with CAD and cerebrovascular disease. ${ }^{15-17}$ Other studies showed genetic evidence of association of insulin or IR with CAD. ${ }^{17-19}$ To confirm and strengthen the emerging association of IR with cardiovascular outcomes, we sought to explore the effects of a recently described multitrait genetic instrument of IR on CAD, myocardial infarction (MI) and ischemic stroke.

MR, using genetic variants as instrumental variables, is a method that can control potential confounding factors that may bias observational studies. ${ }^{12}$ Genetic variants are randomly allocated at meiosis and independent of other factors. Therefore, MR analysis with genetic variants as instrumental variables can prevent confounding and reverse causation, thus make stronger causal inferences between an exposure and risk of diseases. In the present study, we aimed to use MR analysis to determine whether IR is causally associated with cardiovascular diseases, including $\mathrm{CAD}, \mathrm{MI}$, ischemic stroke and its subtypes.

\section{RESEARCH DESIGN AND METHODS}

\section{Study design}

MR analysis was designed to evaluate the causal associations between IR and risk of cardiovascular diseases (figure 1). Genetic variants associated with IR phenotypes were selected as instrumental variable for the MR analysis. We used published summary-level data from four
GWASs of European individuals. ${ }^{20-24}$ Data on the exposure (IR phenotypes) were derived from meta-analysis of GWASs of up to 188577 individuals ${ }^{320-22}$ and data on the outcome (CAD and ischemic stroke) were obtained from GWASs of up to 446696 individuals. ${ }^{23}{ }^{24}$ Characteristics of these GWASs are presented in table 1 and online supplementary methods 1. Analyses of all phenotypes were based on subjects of European ancestry only.

\section{Generation of genetic instrumental variables}

Due to absence of well-powered GWASs for gold standard measures of IR derived from euglycemic clamp and in order to well reflect the underlying phenotype of IR, we used 53 single nucleotide polymorphisms (SNPs) implicated in IR phenotypes identified through meta-analysis of GWASs by Lotta et $a .^{2}{ }^{2}$ Using an integrative genomic approach, Lotta $e t$ al identified 53 SNPs that were associated with three components of IR phenotypes (ie, high fasting insulin, low HDL-C and high TGs) at $\mathrm{p}<0.005$ for each trait in up to 188577 individuals from genome-wide results. ${ }^{2}$ These 53 SNPs were the lead insulin-associated $\mathrm{SNP}$ at each $1 \mathrm{Mb}$ region. Genetic risk score based on these 53 SNPs have been validated to be associated with gold standard measures of IR in independent samples from the Fenland study and four other cohorts. Having a greater number of 53-SNP score was substantially associated with lower insulin sensitivity as measured by euglycemic clamp or insulin suppression test in 2764 individuals $\left(\mathrm{p}=4.3 \times 10^{-6}\right)$ and lower insulin sensitivity index by oral glucose tolerance test in 4769 individuals $\left(\mathrm{p}=7.3 \times 10^{-10}\right){ }^{2}$ The triad of these phenotypes has been proposed as a metric to characterize the genetic architecture of IR. ${ }^{12}$ Summary statistics for association of each SNP with fasting insulin adjusted for body mass index

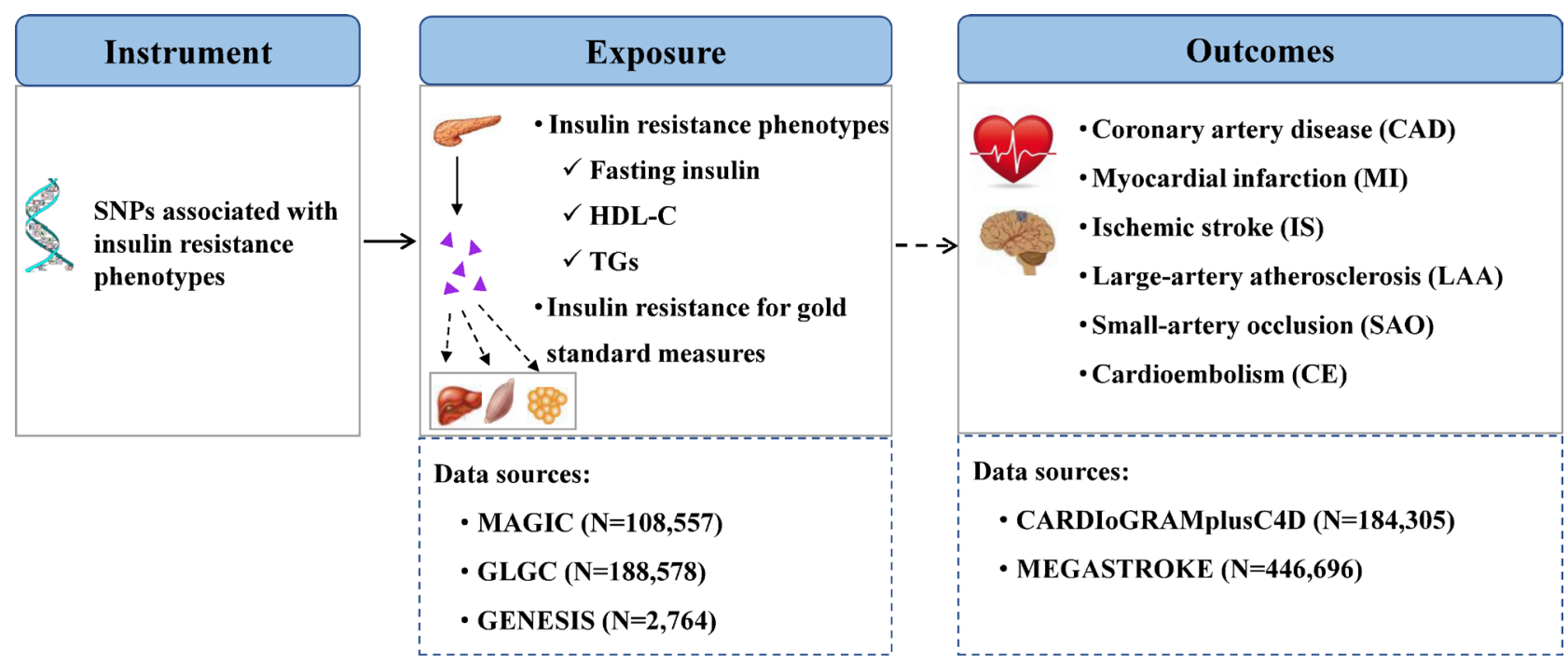

Figure 1 Conceptual framework for the Mendelian randomization analysis of insulin resistance and risk of coronary artery disease and stroke. CARDloGRAMplusC4D, Coronary ARtery Dlsease Genome-wide Replication And Meta-Analysis Plus Coronary Artery Disease Genetics; GENESIS, GENEticS of Insulin Sensitivity; GLGC, Global Lipids Genetics Consortium; HDL-C, high-density lipoprotein cholesterol; MAGIC, Meta-Analyses of Glucose and Insulin-related traits Consortium; MEGASTROKE, Multiancestry Genome-wide Association Study of Stroke; SNP, single nucleotide polymorphisms; TGs, triglycerides. 
Table 1 Characteristics of the GWASs used in this study

\begin{tabular}{|c|c|c|c|c|c|}
\hline Phenotype & Consortium & $\mathbf{N}$ & Ethnicity & Genotype data & PMID \\
\hline \multicolumn{6}{|c|}{ Exposure (insulin resistance phenotypes) ${ }^{*}$} \\
\hline $\begin{array}{l}\text { Fasting insulin } \\
\text { adjusted for BMI }\end{array}$ & MAGIC & Up to 108557 individuals & European & $\begin{array}{l}\text { GWAS array and } \\
\text { metabochip array }\end{array}$ & $\begin{array}{l}22885924 \text { to } \\
22581228\end{array}$ \\
\hline $\begin{array}{l}\mathrm{HDL}-\mathrm{C} \text { and } \\
\text { triglycerides }\end{array}$ & GLGC & Up to 188577 individuals & European & $\begin{array}{l}\text { GWAS array and } \\
\text { metabochip array }\end{array}$ & $\begin{array}{l}24097068 \\
29046328\end{array}$ \\
\hline $\begin{array}{l}\text { Insulin sensitivity } \\
\text { for gold standard } \\
\text { measures }\end{array}$ & GENESIS & 2764 individuals & European & GWAS array & 25798622 \\
\hline \multicolumn{6}{|l|}{ Outcomes } \\
\hline $\begin{array}{l}\text { Coronary artery } \\
\text { disease }\end{array}$ & CARDIoGRAMplusC4D & $\begin{array}{l}\text { Up to } 184305 \text { individuals } \\
\text { (60801 cases and } 123504 \\
\text { controls) }\end{array}$ & European & GWAS array & 26343387 \\
\hline $\begin{array}{l}\text { Myocardial } \\
\text { infarction }\end{array}$ & CARDIoGRAMplusC4D & $\begin{array}{l}\text { Up to } 171876 \text { individuals } \\
\text { (43677 cases and } 128199 \\
\text { controls) }\end{array}$ & European & GWAS array & 26343387 \\
\hline Ischemic stroke & MEGASTROKE & $\begin{array}{l}\text { Up to } 446696 \text { individuals } \\
\text { (40585 cases and } 406111 \\
\text { controls) }\end{array}$ & European & GWAS array & 29531354 \\
\hline $\begin{array}{l}\text { Large-artery } \\
\text { atherosclerosis }\end{array}$ & MEGASTROKE & $\begin{array}{l}\text { Up to } 440328 \text { individuals } \\
\text { (34217 cases and } 406111 \\
\text { controls) }\end{array}$ & European & GWAS array & 29531354 \\
\hline $\begin{array}{l}\text { Small-artery } \\
\text { occlusion }\end{array}$ & MEGASTROKE & $\begin{array}{l}\text { Up to } 411497 \text { individuals } \\
\text { (5386 cases and } 406111 \\
\text { controls) }\end{array}$ & European & GWAS array & 29531354 \\
\hline Cardioembolism & MEGASTROKE & $\begin{array}{l}\text { Up to } 413304 \text { individuals } \\
\text { (7193 cases and } 406111 \\
\text { controls) }\end{array}$ & European & GWAS array & 29531354 \\
\hline
\end{tabular}

*Lotta et $\mathrm{al}^{2}$ identified 53 genetic variants for insulin resistance phenotypes by combining published GWAS results for fasting insulin adjusted for BMI, HDL-C and triglycerides, and Wang et al ${ }^{3}$ generated a composite genetic instrument for insulin resistance phenotypes by meta-analysis of these genetic variants.

BMI, body mass index; CARDloGRAMplusC4D, Coronary ARtery Dlsease Genome-wide Replication And Meta-Analysis Plus Coronary Artery Disease Genetics; GENESIS, GENEticS of Insulin Sensitivity; GLGC, Global Lipids Genetics Consortium; GWAS, genome-wide association study; HDL-C, high-density lipoprotein cholesterol; MAGIC, Meta-Analyses of Glucose and Insulin-related traits Consortium; MEGASTROKE, Multiancestry Genome-wide Association Study of Stroke; PMID, PubMed unique identifier.

(BMI) were acquired from the Meta-Analyses of Glucose and Insulin-related traits Consortium (MAGIC) ${ }^{20} 21$ and that with HDL-C or TGs levels from the Global Lipids Genetics Consortium (GLGC). ${ }^{22}$ A subset 25 of these 53 loci had previously been associated with HDL-C or TGs levels at genome-wide significance, whereas 28 had not. ${ }^{2}$ All the SNPs were in different genomic regions and in linkage equilibrium (online supplementary table 1). Potential pleiotropic effects (whether the genetic variants affect the outcome independently of the exposure of interest) of these SNPs were assessed through the MR-Egger regression method. The slope of the MR-Egger regression represents pleiotropy-corrected causal estimates and the intercept represents the average pleiotropic effects across all SNPs.

As Lotta $e t a l^{2}$ did not provide beta-coefficient and SE for the association of these individual SNPs with the IR phenotype, we used the composite genetic instrument for IR phenotypes generated based on these 53 SNPs estimates by Wang et $a l^{3}$ An estimate of each of the 53 SNP associations with the composite IR phenotypes were generated through meta-analysis of the absolute values of the standardized beta-coefficient for each SNP association with the individual components of IR phenotypes (ie, high fasting insulin adjusted for BMI, low HDL-C and high TGs) using a fixed-effect inverse-variance weighted (IVW) method. ${ }^{3}$ We used this meta-analyzed value as the SNP-exposure (IR phenotypes) estimate (online supplementary table 1); 1-SD genetically higher IR phenotypes was associated with $55 \%$ higher fasting insulin adjusted for BMI, $0.46 \mathrm{mmol} / \mathrm{L}$ lower HDL-C and $0.89 \mathrm{mmol} / \mathrm{L}$ higher TGs. ${ }^{3}$

As Wang $e t a l^{3}$ reported, most of the SNPs had a similar contribution of the three traits to the composite IR phenotypes with the exception of the SNP rs1011685 (near $L P L)$, which had a much weaker effect on insulin adjusted for BMI. The heterogeneity of association between the composite IR phenotypes and the three traits was substantially reduced after exclusion of rs1011685 (for insulin: $\mathrm{Q}=235.29, \mathrm{p}<0.001, \mathrm{I}^{2}=78 \%$ to $\mathrm{Q}=49.71, \mathrm{p}=0.52$, $\mathrm{I}^{2}=0 \%$; for HDL-C: $\mathrm{Q}=73.76, \mathrm{p}=0.03, \mathrm{I}^{2}=30 \%$ to $\mathrm{Q}=57.25$, $\mathrm{p}=0.25, \mathrm{I}^{2}=11 \%$; for TGs: $\mathrm{Q}=139.17, \mathrm{p}<0.001, \mathrm{I}^{2}=63 \%$ to 
$\left.\mathrm{Q}=57.19, \mathrm{p}=0.26, \mathrm{I}^{2}=11 \%\right)$. Therefore, sensitivity analyses were conducted after exclusion of rs1011685 from the instrument. ${ }^{3}$

\section{Outcomes}

Summary statistics for the association of each SNP with $\mathrm{CAD}$ and $\mathrm{MI}$ were acquired from the previously published Coronary ARtery DIsease Genome-wide Replication And Meta-Analysis Plus Coronary Artery Disease Genetics (CARDIoGRAMplusC4D) 1000 Genomes-based GWAS, ${ }^{23}$ and that with ischemic stroke as a whole and the three main subtypes (large-artery atherosclerosis (LAA), small-artery occlusion (SAO), cardioembolism (CE)) from the previously published GWAS of Multiancestry Genome-wide Association Study of Stroke (MEGASTROKE) consortium, ${ }^{24}$ respectively (table 1 and online supplementary method 1). The associations of the 53 individual SNPs for the IR phenotypes with CAD and MI, and ischemic stroke and its subtypes are presented in online supplementary tables 2 and 3, respectively.

\section{Statistical analysis}

The SNP-IR phenotypes and SNP-outcome associations were used to compute estimates of IR phenotypesoutcome associations using MR analyses. We used a conventional IVW MR analysis in which the SNP-outcome estimate is regressed on the SNP-IR phenotypes estimate, weighted by the inverse-variance of SNP-outcome estimate and with the $y$-axis intercept is fixed to zero. ${ }^{25}$ The IVW estimate is an efficient analysis method when all genetic variants are valid instruments. In sensitivity analyses, we also conducted MR-Egger, simple median, weighted-median methods of MR analyses, which are more robust to the inclusion of pleiotropic instruments. The MR-Egger method can identify and control for bias due to directional pleiotropy (ie, whether causal estimates from weaker variants tend to be skewed in one direction) and provide an effect estimate which is not subject to some violations of the standard instrumental variable assumptions. ${ }^{26}$ The slope of the MR-Egger regression can provide pleiotropy-corrected causal estimates. An $I_{G X}^{2}$ statistic was also calculated to test the presence of measurement error in MR-Egger results; $I_{G X}^{2}$ statistic $>0.90$ was considered no obvious violation of 'No Measurement Error' assumption. ${ }^{27}$ The weighted-median method can provide a consistent estimate of the causal effect even when up to $50 \%$ of the information contributing to the analysis comes from genetic variants that are invalid instruments. $^{28}$ These approaches may assess the robustness of estimates to potential violations of the instrumental variable assumptions.

In addition to the 53-SNPs instruments, we also conducted sensitivity analyses based on: 1) 52-SNPs instruments with the exclusion of rs1011685 (near LPL), which as described above, did not show consistent associations across individual phenotypes of IR; 2) 28-SNPs instruments reported in Lotta et $a l^{2}$ that were not in loci previously associated with HDL-C or TGs at genome-wide significance; 3) 44-SNPs instruments after exclusion of 9 SNPs individually associated with BMI at $\mathrm{p}<0.001 \mathrm{using}$ Genetic Investigation of ANthropometric Traits summary statistics from 53-SNPs instrument identified by Lotta et $a l^{2329}$; 4) $12-$ SNPs instruments reported by the MAGIC investigators that were associated with fasting insulin (BMI adjusted) at genome-wide significance (online supplementary table 4) ${ }^{20}$; 5) 5-SNPs instruments for gold standard measures of IR, such as euglycemic clamp or insulin suppression test, identified by GENEticS of Insulin Sensitivity consortium through GWAS in 2764 European individuals (online supplementary table 4)..$^{30}$

Genetic effect estimates of the exposure-outcome associations are presented as OR with their 95\% CI of outcome (CAD, MI, ischemic stroke and its subtypes) per 1-SD genetically higher IR phenotypes. To gain insight into the association of the composite genetic IR phenotypes with its individual components and the outcomes, we quantified the association of a 1-SD higher genetically elevated IR phenotypes on the individual components of IR phenotypes (fasting insulin adjusted for BMI, HDL-C and TGs) and the outcomes (CAD, MI, ischemic stroke and its subtypes). To ensure the validity of our conclusions, we took a conservative approach and applied a Bonferroni-corrected significance threshold calculated as 0.05 divided by 6 (ie, $0.0083 ; 0.05 / 6$ for six outcomes). We considered a statistical test with an observed twosided $\mathrm{p}$ value $<0.05$ as nominally significant evidence for a potential, but yet to be confirmed, causal association; and an observed two-sided $\mathrm{p}$ value $<0.0083$ as statistically significant evidence for a causal association. ${ }^{31}$ All analyses were conducted with R V.3.5.1 (R Development Core Team).

\section{RESULTS}

\section{Causal association of IR with CAD}

The IVW method showed that 1-SD increase in IR phenotypes was causally associated with a substantial increase in risk of $\mathrm{CAD}(\mathrm{OR}=1.79,95 \% \mathrm{CI}: 1.57$ to $2.04, \mathrm{p}<0.001)$ and $\mathrm{MI}(\mathrm{OR}=1.78,95 \% \mathrm{CI}: 1.54$ to $2.06, \mathrm{p}<0.001)$ at the Bonferroni-adjusted level of significance $(\mathrm{p}<0.0083)$ using the 53-SNPs instrument (figure 2). MR-Egger regression showed no evidence of directional pleiotropy for the association of IR phenotypes with CAD (intercept=0.002, $\mathrm{p}=0.67$ ) and $\mathrm{MI}$ (intercept=0.000, $\mathrm{p}=0.98$ ) (table 2). Similar magnitudes of association and no evidence of directional pleiotropy were observed using the 52-SNPs and 28-SNPs instruments. There was a low risk of bias with MR-Egger because of measurement error using 53-SNPs instrument $\left(I_{G X}^{2}\right.$ statistic $=94.6 \%$ for CAD and $94.6 \%$ for MI) but not using 28-SNPs and 52-SNPs instrument $\left(I_{G X}^{2}\right.$ statistic $=9.6 \%$ and $78.9 \%$ for CAD, $8.3 \%$ and $79.5 \%$ for MI). Associations between each variant with IR phenotypes and risk of CAD and MI are displayed in figure 3 and online supplementary figure 1 .

In sensitivity analyses using the simple median and weighted-median method of MR analyses, similar 


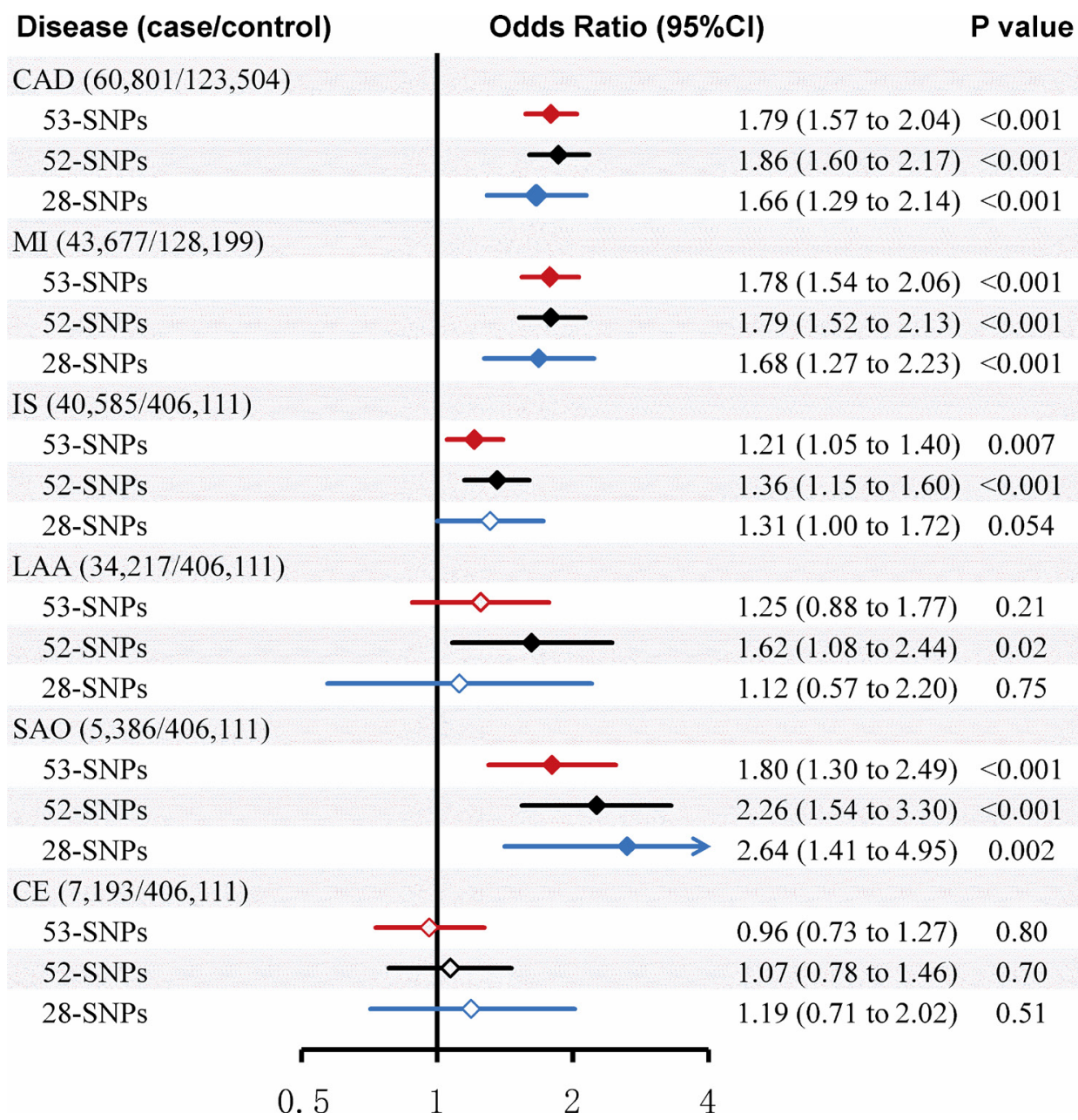

Figure 2 Causal effect estimates of genetically predicted insulin resistance phenotypes on coronary artery disease and ischemic stroke. Estimates are derived from inverse-variance weighted method of Mendelian randomization analysis and represented OR $(95 \% \mathrm{Cl})$ per $1-\mathrm{SD}$ insulin resistance phenotypes. Open and closed symbols indicate $\mathrm{p} \geq 0.05$ and $\mathrm{p}<0.05$, respectively. CAD, coronary artery disease; CE, cardioembolism; IS, ischemic stroke; LAA, large-artery atherosclerosis; MI, myocardial infarction; SAO, small-artery occlusion; SNP, single nucleotide polymorphism.

association were observed using 53-SNPs, 52-SNPs and 28-SNPs instruments (table 2). However, nominal associations using the 52-SNPs instruments $(\mathrm{p}=0.01, \mathrm{p}=0.045)$, but no significant association using 28-SNPs instrument $(\mathrm{p}=0.92, \mathrm{p}=0.74)$, were observed both for the risk of CAD and MI using MR-Egger regression method. Further sensitivity analysis using 44-SNPs instruments that were not associated with BMI at $\mathrm{p}<0.001$ and 12-SNPs instruments that were associated with fasting insulin (BMI adjusted) at genome-wide significance showed significant association of IR phenotypes with the risk of CAD and MI (all $\mathrm{p}<0.001$; online supplementary figure 2). However, association was not observed for CAD or MI using 5-SNPs instruments for gold standard measures of IR $(\mathrm{p}=0.17$; $\mathrm{p}=0.12)$.

\section{Causal association of IR with ischemic stroke}

The IVW method showed that 1-SD increase in IR phenotypes was causally associated with a substantial increase in risk of ischemic stroke $(\mathrm{OR}=1.21,95 \%$ CI: 1.05 to $1.40, \mathrm{p}=0.007)$ and the $\mathrm{SAO}$ subtype of stroke $(\mathrm{OR}=1.80$,
95\% CI: 1.30 to $2.49, \mathrm{p}<0.001)$ at the Bonferroniadjusted level of significance $(\mathrm{p}<0.0083)$, but no significant association for the LAA (OR=1.25, 95\% CI: 0.88 to 1.77, $\mathrm{p}=0.21)$ and $\mathrm{CE}(\mathrm{OR}=0.96,95 \% \mathrm{CI}: 0.73$ to 1.27 , $\mathrm{p}=0.80$ ) subtypes of stroke using the 53-SNPs instrument (figure 2). MR-Egger regression showed no evidence of directional pleiotropy for the associations of IR phenotypes with ischemic stroke (intercept $=0.007, \mathrm{p}=0.053$ ), LAA (intercept $=0.006, p=0.48$ ) and $\mathrm{CE}$ subtypes (intercept $=0.008, p=0.24)$, but marginal significant for the SAO subtype (intercept $=0.017, \mathrm{p}=0.046$ ) (table 2). Similar magnitudes of association and no evidence of directional pleiotropy were observed using the 52-SNPs and 28-SNPs instruments. Additionally, nominal association were observed between 1-SD increase in IR phenotypes and risk of the LAA subtype (OR=1.62, 95\% CI: 1.08 to 2.44, $\mathrm{p}=0.02$ ) using 52-SNPs instrument. There was a low risk of bias with MR-Egger because of measurement error using 53-SNPs instrument $\left(I_{G X}^{2}\right.$ statistic $=94.7 \%, 94.8 \%$, $94.7 \%$ and $94.7 \%$ for IS, LAA, SAO and CE, respectively) 
Table 2 MR statistical sensitivity analyses

\begin{tabular}{|c|c|c|c|c|c|c|c|c|c|}
\hline \multirow[b]{2}{*}{$\begin{array}{l}\text { Outcome (case/ } \\
\text { control) }\end{array}$} & \multicolumn{5}{|l|}{ MR-Egger } & \multicolumn{2}{|c|}{ Simple median } & \multicolumn{2}{|c|}{ Weighted-median } \\
\hline & OR $(95 \% \mathrm{Cl})$ & $P$ value & Intercept $(95 \% \mathrm{CI})$ & $\begin{array}{l}\text { P value for } \\
\text { intercept }\end{array}$ & $I_{G X}^{2}$ & OR (95\% Cl) & $P$ value & OR $(95 \% \mathrm{Cl})$ & $P$ value \\
\hline \multicolumn{10}{|c|}{ CAD (60 801/123 504) } \\
\hline 53-SNPs & $\begin{array}{l}1.68(1.22 \text { to } \\
2.32)\end{array}$ & 0.002 & $\begin{array}{l}0.002(-0.006 \text { to } \\
0.009)\end{array}$ & 0.67 & $94.6 \%$ & $\begin{array}{l}2.02(1.60 \text { to } \\
2.54)\end{array}$ & $<0.001$ & $\begin{array}{l}1.73(1.39 \text { to } \\
2.16)\end{array}$ & $<0.001$ \\
\hline 52-SNPs & $\begin{array}{l}2.12 \text { (1.18 to } \\
3.82)\end{array}$ & 0.01 & $\begin{array}{l}-0.003(-0.014 \text { to } \\
0.009)\end{array}$ & 0.65 & $78.9 \%$ & $\begin{array}{l}2.03 \text { ( } 1.59 \text { to } \\
2.57)\end{array}$ & $<0.001$ & $\begin{array}{l}2.09 \text { (1.66 to } \\
2.64)\end{array}$ & $<0.001$ \\
\hline 28-SNPs & $\begin{array}{l}0.94 \text { ( } 0.30 \text { to } \\
2.99)\end{array}$ & 0.92 & $\begin{array}{l}0.009(-0.009 \text { to } \\
0.028)\end{array}$ & 0.32 & $9.6 \%$ & $\begin{array}{l}1.97 \text { (1.37 to } \\
2.83)\end{array}$ & $<0.001$ & $\begin{array}{l}1.86(1.30 \text { to } \\
2.67)\end{array}$ & 0.001 \\
\hline \multicolumn{10}{|c|}{ MI (43 677/128 199) } \\
\hline 53-SNPs & $\begin{array}{l}1.78 \text { (1.27 to } \\
2.49)\end{array}$ & 0.001 & $\begin{array}{l}0.000(-0.007 \text { to } \\
0.008)\end{array}$ & 0.98 & $94.6 \%$ & $\begin{array}{l}1.74 \text { (1.36 to } \\
2.23)\end{array}$ & $<0.001$ & $\begin{array}{l}1.76 \text { (1.35 to } \\
2.30)\end{array}$ & $<0.001$ \\
\hline 52-SNPs & $\begin{array}{l}1.87 \text { (1.01 to } \\
3.44)\end{array}$ & 0.045 & $\begin{array}{l}-0.001(-0.013 \text { to } \\
0.011)\end{array}$ & 0.89 & $79.5 \%$ & $\begin{array}{l}1.76 \text { ( } 1.35 \text { to } \\
2.29)\end{array}$ & $<0.001$ & $\begin{array}{l}1.97 \text { (1.52 to } \\
2.56)\end{array}$ & $<0.001$ \\
\hline 28-SNPs & $\begin{array}{l}0.80(0.20 \text { to } \\
3.13)\end{array}$ & 0.74 & $\begin{array}{l}0.012(-0.010 \text { to } \\
0.034)\end{array}$ & 0.27 & $8.3 \%$ & $\begin{array}{l}2.02(1.34 \text { to } \\
3.06)\end{array}$ & 0.001 & $\begin{array}{l}1.99 \text { (1.32 to } \\
3.02)\end{array}$ & 0.001 \\
\hline \multicolumn{10}{|c|}{ IS (40 585/406 111) } \\
\hline 53-SNPs & $\begin{array}{l}0.92 \text { (0.66 to } \\
1.27)\end{array}$ & 0.61 & $\begin{array}{l}0.007 \text { (0.000 to } \\
0.053)\end{array}$ & 0.053 & $94.7 \%$ & $\begin{array}{l}1.39 \text { (1.09 to } \\
1.78)\end{array}$ & 0.009 & $\begin{array}{l}1.15 \text { (0.92 to } \\
1.44)\end{array}$ & 0.21 \\
\hline 52-SNPs & $\begin{array}{l}1.32 \text { ( } 0.71 \text { to } \\
2.44)\end{array}$ & 0.38 & $\begin{array}{l}0.001(-0.011 \text { to } \\
0.012)\end{array}$ & 0.92 & $78.8 \%$ & $\begin{array}{l}1.39 \text { (1.09 to } \\
1.78)\end{array}$ & 0.009 & $\begin{array}{l}1.39 \text { (1.09 to } \\
1.77)\end{array}$ & 0.008 \\
\hline 28-SNPs & $\begin{array}{l}2.07(0.41 \text { to } \\
10.42)\end{array}$ & 0.38 & $\begin{array}{l}-0.007(-0.033 \text { to } \\
0.018)\end{array}$ & 0.57 & $0.0 \%$ & $\begin{array}{l}1.55 \text { (1.02 to } \\
2.36)\end{array}$ & 0.04 & $\begin{array}{l}1.63 \text { (1.08 to } \\
2.48)\end{array}$ & 0.02 \\
\hline \multicolumn{10}{|c|}{ LAA (34 217/406 111) } \\
\hline 53-SNPs & $\begin{array}{l}0.97 \text { (0.44 to } \\
2.16)\end{array}$ & 0.94 & $\begin{array}{l}0.006(-0.011 \text { to } \\
0.024)\end{array}$ & 0.48 & $94.8 \%$ & $\begin{array}{l}1.33(0.72 \text { to } \\
2.44)\end{array}$ & 0.36 & $\begin{array}{l}1.00 \text { (0.59 to } \\
1.72)\end{array}$ & 0.99 \\
\hline 52-SNPs & $\begin{array}{l}5.69(1.30 \text { to } \\
24.78)\end{array}$ & 0.02 & $\begin{array}{l}-0.025(-0.053 \text { to } \\
0.003)\end{array}$ & 0.08 & $79.1 \%$ & $\begin{array}{l}1.33(0.73 \text { to } \\
2.44)\end{array}$ & 0.36 & $\begin{array}{l}1.86(1.01 \text { to } \\
3.40)\end{array}$ & 0.046 \\
\hline 28-SNPs & $\begin{array}{l}6.03(0.17 \text { to } \\
216.59)\end{array}$ & 0.33 & $\begin{array}{l}-0.027(-0.084 \text { to } \\
0.029)\end{array}$ & 0.34 & $0.0 \%$ & $\begin{array}{l}1.03(0.38 \text { to } \\
2.78)\end{array}$ & 0.95 & $\begin{array}{l}0.90 \text { (0.33 to } \\
2.44)\end{array}$ & 0.84 \\
\hline \multicolumn{10}{|c|}{ SAO $(5386 / 406$ 111) } \\
\hline 53-SNPs & $\begin{array}{l}0.94 \text { (0.45 to } \\
1.96)\end{array}$ & 0.87 & $\begin{array}{l}0.017 \text { (0.000 to } \\
0.033)\end{array}$ & 0.046 & $94.7 \%$ & $\begin{array}{l}2.28 \text { (1.28 to } \\
4.08)\end{array}$ & 0.005 & $\begin{array}{l}1.09 \text { (0.66 to } \\
1.81)\end{array}$ & 0.74 \\
\hline 52-SNPs & $\begin{array}{l}1.49(0.35 \text { to } \\
6.27)\end{array}$ & 0.59 & $\begin{array}{l}0.008(-0.019 \text { to } \\
0.036)\end{array}$ & 0.55 & $78.8 \%$ & $\begin{array}{l}2.56 \text { (1.44 to } \\
4.53)\end{array}$ & 0.001 & $\begin{array}{l}2.28 \text { (1.28 to } \\
4.04)\end{array}$ & 0.005 \\
\hline 28-SNPs & $\begin{array}{l}14.43(0.58 \text { to } \\
359.96)\end{array}$ & 0.10 & $\begin{array}{l}-0.028(-0.078 \text { to } \\
0.023)\end{array}$ & 0.29 & $0.0 \%$ & $\begin{array}{l}2.28 \text { (0.90 to } \\
5.75)\end{array}$ & 0.08 & $\begin{array}{l}2.24 \text { (0.89 to } \\
5.64)\end{array}$ & 0.09 \\
\hline \multicolumn{10}{|l|}{ CE (7193/406 111) } \\
\hline 53-SNPs & $\begin{array}{l}0.71 \text { ( } 0.39 \text { to } \\
1.28)\end{array}$ & 0.25 & $\begin{array}{l}0.008(-0.005 \text { to } \\
0.021)\end{array}$ & 0.24 & $94.7 \%$ & $\begin{array}{l}0.96 \text { (0.61 to } \\
1.51)\end{array}$ & 0.86 & $\begin{array}{l}0.72 \text { (0.46 to } \\
1.14)\end{array}$ & 0.16 \\
\hline 52-SNPs & $\begin{array}{l}0.82(0.26 \text { to } \\
2.60)\end{array}$ & 0.74 & $\begin{array}{l}0.005 \text { ( }-0.017 \text { to } \\
0.027)\end{array}$ & 0.65 & $79.2 \%$ & $\begin{array}{l}0.97 \text { ( } 0.61 \text { to } \\
1.55)\end{array}$ & 0.90 & $\begin{array}{l}1.12(0.70 \text { to } \\
1.78)\end{array}$ & 0.64 \\
\hline 28-SNPs & $\begin{array}{l}0.95(0.05 \text { to } \\
19.85)\end{array}$ & 0.97 & $\begin{array}{l}0.004(-0.044 \text { to } \\
0.052)\end{array}$ & 0.88 & $0.0 \%$ & $\begin{array}{l}0.97 \text { ( } 0.45 \text { to } \\
2.11)\end{array}$ & 0.94 & $\begin{array}{l}0.98 \text { ( } 0.45 \text { to } \\
2.13)\end{array}$ & 0.96 \\
\hline
\end{tabular}

CAD, coronary artery disease; CE, cardioembolism; IS, ischemic stroke; LAA, large-artery atherosclerosis; MI, myocardial infarction; MR, Mendelian randomization; SAO, small-artery occlusion; SNP, single nucleotide polymorphism.

but not using 28-SNPs and 52-SNPs instrument $\left(I_{G X}^{2}\right.$ statistic $=0.0 \%$ using 28 -SNPs instrument; $78.8 \%, 79.1 \%$, $78.8 \%$ and $79.2 \%$ for IS, LAA, SAO and CE, respectively using 52-SNPs instrument). Associations between each variant with IR phenotypes and risk of ischemic stroke, the LAA and SAO subtypes of stroke are displayed in figure 3 and online supplementary figure 1 .
In sensitivity analyses, significant association was observed for risk of ischemic stroke using weightedmedian method with 52-SNPs instrument $(\mathrm{p}=0.008)$. Nominal associations were observed for the risk of ischemic stroke using the simple median with 53-SNPs $(\mathrm{p}=0.009)$, 52-SNPs $(\mathrm{p}=0.009)$ and 28-SNPs $(\mathrm{p}=0.04)$ instruments and weighted-median method with 28-SNPs 
A

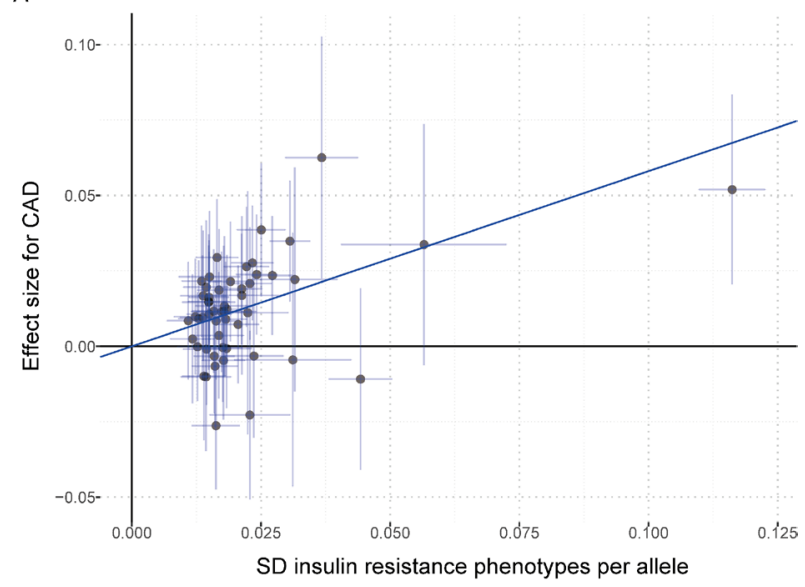

B

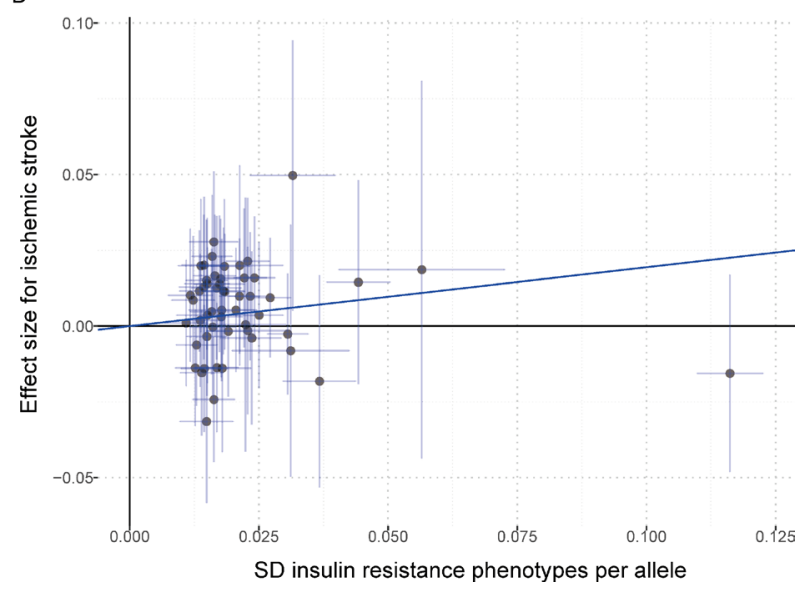

Figure 3 Associations of IR phenotypes variants with risk of CAD (A) and ischemic stroke (B). The blue line indicates the estimate of effect using inverse-variance weighted method. Circles indicate marginal genetic associations with IR phenotypes and risk of outcome for each variant. Error bars indicate $95 \% \mathrm{Cls}$. CAD, coronary artery disease; IR, insulin resistance.

( $\mathrm{p}=0.02)$ instruments, and for the risk of the LAA subtype using MR-Egger regression and weighted median methods with the 52-SNPs instrument $(\mathrm{p}=0.02 ; \mathrm{p}=0.046)$ (table 2). Significant associations were observed for the risk of the SAO subtype using the simple median method with the 53-SNPs and 52-SNPs instruments $(\mathrm{p}=0.005 ; \mathrm{p}=0.001)$ and the weighted-median method with the 52-SNPs instrument $(\mathrm{p}=0.005)$. Further sensitivity analysis using 44-SNPs instruments that were not associated with BMI at $\mathrm{p}<0.001$ showed significant association of IR phenotypes with ischemic stroke $(\mathrm{p}=0.008)$ and nominal association with SAO $(\mathrm{p}=0.009)$ (online supplementary figure 2). Using 12-SNPs instruments that were associated with fasting insulin (BMI adjusted) at genome-wide significance and 5-SNPs instruments for gold standard measures of IR, nominal associations were observed for SAO ( $\mathrm{p}=0.02$ and $\mathrm{p}=0.01$ ). No significant association was observed in analyses using other methods or instruments.

\section{DISCUSSION}

Using MR analysis, our study provides genetic evidence in support that higher level of IR may lead to increased risk of cardiovascular diseases. In this study, genetically predicted higher level of IR phenotypes was associated with an increased risk of CAD, MI, ischemic stroke and the SAO subtype of stroke. Higher level of IR phenotypes was potentially, yet to be confirmed, causally associated with an increased risk of the LAA subtype of stroke. However, no significant association was observed between IR phenotypes and risk of the CE subtype of stroke.

The findings were consistent with previous observational studies showing a positive association of IR with risk of CAD. ${ }^{6} 7$ Our results also were consistent with previous MR analysis based on the Finnish dataset that revealed causal effects between glycemic traits (insulin and glucose) and coronary heart disease. ${ }^{19}$ However, results from the Northern Manhattan Study showed that IR was associated with risk of combined outcomes (ischemic stroke, MI and vascular death) after controlling for demographic factors but was attenuated and no longer significant after controlling for metabolic syndrome status or after adjustment for vascular risk factors. ${ }^{9}$ Women's Health Initiative Biomarkers studies also implicated that IR measures were no longer associated with cardiovascular risk after adjustment for HDL-C in postmenopausal women without diabetes mellitus. ${ }^{8}$ The reason for the discrepancy between our study and these studies is unclear. The potential explanation might be that the above-mentioned observational studies were overadjusted since metabolic syndrome and high HDL-C were considered as pathophysiological consequences or traits of IR. ${ }^{5}$ MR associations were attenuated or abolished after using the 28-SNPs instruments which excluded SNPs that had been associated with HDL-C or TGs (a similar adjustment for HDL-C and TGs), indicating that the major contribution of the IR composite phenotype to the $\mathrm{CAD} / \mathrm{MI}$ outcome is via its effect on lipids. This may also due to the quality of this analysis given the low $I_{G X}^{2}$ statistic values. Attenuated associations were not observed in MR analyses using 44-SNPs instrument which excluded SNPs that were associated with BMI, indicating that the association of IR and cardiovascular events were not mainly mediated by BMI. These results suggest that a composite assessment of IR phenotypes that includes HDL-C and TGs is a better proxy of IR and predictor of cardiovascular outcomes.

Our study also observed that genetically predicted IR phenotypes was positively associated with an increased risk of ischemic stroke, substantially the SAO subtype and potentially the LAA subtype of stroke. These results were consistent with previous observational studies. ${ }^{9} 1032$ Both observational results from the Northern Manhattan Study ${ }^{9}$ and the Cardiovascular Health Study ${ }^{10}$ showed that IR were associated with increased risk of incident ischemic stroke in non-diabetic populations. Results from the REasons for Geographic And Racial Differences in Stroke Study indicated a marginal positive association of 
IR with risk of ischemic stroke in white population but no association in blacks. ${ }^{32}$ However, the association was not validated in the Rotterdam Study. ${ }^{11}$ In contrast, studies of the association between IR and risk of etiological subtypes of ischemic stroke are limited. IR was showed to be associated with intracranial and carotid atherosclerosis but can be largely explained by the clustered expression of components of the metabolic syndrome. ${ }^{33}{ }^{34} \mathrm{~A}$ cross-sectional study in Korea showed that IR was an independent risk factor of silent lacunar infarction presence and its severity. ${ }^{35}$ The present study adds the evidence of causal impact of genetic-predicted IR phenotypes and risk of ischemic stroke and its subtypes using MR analysis, a method that may control unmeasured confounding factors and its potential to ascertain causal relationship. ${ }^{36}$ For ischemic stroke and SAO subtype, the weightedmedian method with 53-SNPs instruments completely attenuated the significance, but the 52-SNPs instruments which removed the outlying SNP rs1011685 recovered this. This may be because the results of weighted-median method with 53-SNPs instruments were much driven by the SNP rs1011685, which had a negative association with the outcomes but large weight in weighted-median method.

IR results from defective intracellular signaling that affects glucose transport. The pathophysiological consequences of IR include hypertension, dyslipidemia, abnormal fibrinolysis, hyperglycemia, hyperinsulinemia, systemic inflammation, altered vascular endothelial function and atherogenesis. ${ }^{5}$ Recent MR analysis based on GWASs showed that IR causally affects all branched-chain amino acids (isoleucine, leucine, valine) and inflammation, whose metabolism lie on a causal pathway from IR to type 2 diabetes. ${ }^{3}$ These metabolic and cellular changes may then promote atherosclerosis and subsequent clinical events, including CAD and ischemic stroke. ${ }^{5}$ Using genetic data via an MR approach, we assessed the causal relationship between IR phenotypes and risk of cardiovascular diseases. The results showed that genetic predisposition to IR phenotypes were related to higher risk of CAD, MI, ischemic stroke and the SAO subtype of stroke, and potentially the LAA subtype of stroke.

Strengths of our study is the design of MR analysis based on large-scale GWASs using multiple IR phenotypesrelated SNPs, which enable us to perform a comprehensive evaluation of IR and increase the precision of the estimates. The design of MR analysis can prevent reverse causation and potential confounders, such as dietary and lifestyle preference, thus ascertain causal inferences. ${ }^{12}$ Our analysis distinguishes itself from previous MR study ${ }^{19}$ by performing a comprehensive evaluation of causal associations of IR with risk of CAD and ischemic stroke as well as its subtypes. Comprehensive evaluation of subsequent clinical events with $\mathrm{CAD}$ and stroke may help better understanding of the clinical consequences of IR.

Our study had several limitations. First, our analyses were conducted using European datasets and generalization of the findings to population of non-European ancestry was limited. However, recent studies are providing evidence of shared genetic architecture for metabolic diseases between Europeans and nonEuropeans. ${ }^{37}$ The uniformity of the included subjects ensures minimal risk of confounding by population admixture. Second, the identification of IR phenotype was through proxy IR based on a meta-GWAS of three traits (higher fasting insulin levels adjusted for BMI, lower HDL-C and higher TGs levels). As we known, the 'gold standard' for quantifying IR is the euglycemic hyperinsulinemic glucose clamp technique. Due to lack of data regarding large-scale GWAS on gold standard measures of insulin sensitivity and in order to well reflect the underlying phenotype of IR, we used this proxy measure of IR with SNP-phenotype associations at $\mathrm{p}<0.005$ for each of the three traits. The selection condition with $\mathrm{p}<0.005$ creates a concordance of all three by selection rather than biology and the proxy measure of IR might just represent a very specific weighted sum of the three traits. This may cause misclassification bias. However, the identified loci were strongly associated with risk of diabetes and gold standard measures of insulin sensitivity in the validation population in the original paper by Lotta $e t a l .^{2}$ Third, there was a risk of bias because of measurement error using 28-SNPs and 52-SNPs instrument and the results may be biased by potential pleiotropy (SNPs may tag heterogeneous pathways) since we used MR design. ${ }^{38}$ Caution is needed to explain the results as no significant association was observed either for the risk of CAD or MI using 28-SNPs instrument and MR-Egger regression method, which may provide pleiotropy-corrected causal estimates. ${ }^{26}$ However, pleiotropic effects were not observed in MR-Egger regression analyses and sensitivity analyses with exclusion of non-specific SNPs showed mostly similar results. Finally, sample size of GWAS for stroke subtypes was limited and the causal inferences of IR phenotypes and stroke subtypes need further validation based on GWASs with larger sample sizes.

\section{CONCLUSIONS}

Our MR analysis provide new evidences of causal associations between IR and risk of cardiovascular diseases, especially for the risk of CAD, MI, ischemic stroke and the SAO subtype of stroke. However, further validations are needed in other studies with large sample sizes for the risk of stroke subtypes.

Acknowledgements Data on glycemic traits have been contributed by MAGIC investigators. Data on lipid traits have been contributed by GLGC investigators. Data on coronary artery disease/myocardial infarction have been contributed by CARDIoGRAMplusC4D investigators. Data on stroke have been contributed by MEGASTROKE investigators.

Contributors WC and YP designed the study and drafted the manuscript. SW, WL and YP collected the data. WC and YP performed the analysis and interpreted the data.

Funding This work was supported by grants from the National Natural Science Foundation of China (81971091, 81901177), Beijing Hospitals Authority Youth 
Programme (QML20190501), Ministry of Science and Technology of the People's Republic of China (2016YFC0901002, 2016YFC0901001, 2017YFC1310901, 2017YFC1310902, 2017YFC1307905, 2018YFC1311700 and 2018YFC1311706), Beijing Municipal Administration of Hospitals (SML20150502), Beijing Municipal Science \& Technology Commission (D171100003017002, D151100002015003), National Science and Technology Major Project (2017ZX09304018) and Beijing Tiantan Hospital (2018-YQN-1). The MEGASTROKE project received funding from sources specified at http://www.megastroke.org/acknowledgments.html.

Competing interests None declared.

Patient consent for publication Not required.

Ethics approval The protocol and data collection were approved by the ethics committee of the original GWAS study sites.

Provenance and peer review Not commissioned; externally peer reviewed.

Data availability statement All data relevant to the study are included in the article or uploaded as supplementary information. There are no additional, unpublished data available from this study.

Open access This is an open access article distributed in accordance with the Creative Commons Attribution Non Commercial (CC BY-NC 4.0) license, which permits others to distribute, remix, adapt, build upon this work non-commercially, and license their derivative works on different terms, provided the original work is properly cited, appropriate credit is given, any changes made indicated, and the use is non-commercial. See: http://creativecommons.org/licenses/by-nc/4.0/.

ORCID iD

Yuesong Pan http://orcid.org/0000-0003-3082-6789

\section{REFERENCES}

1 Scott RA, Fall T, Pasko D, et al. Common genetic variants highlight the role of insulin resistance and body fat distribution in type 2 diabetes, independent of obesity. Diabetes 2014;63:4378-87.

2 Lotta LA, Gulati P, Day FR, et al. Integrative genomic analysis implicates limited peripheral adipose storage capacity in the pathogenesis of human insulin resistance. Nat Genet 2017;49:17-26.

3 Wang Q, Holmes MV, Davey Smith G, et al. Genetic support for a causal role of insulin resistance on circulating branched-chain amino acids and inflammation. Diabetes Care 2017:40:1779-86.

4 Ginsberg HN. Insulin resistance and cardiovascular disease. J Clin Invest 2000;106:453-8.

5 Kernan WN, Inzucchi SE, Viscoli CM, et al. Insulin resistance and risk for stroke. Neurology 2002;59:809-15.

6 Saely $\mathrm{CH}$, Aczel S, Marte T, et al. The metabolic syndrome, insulin resistance, and cardiovascular risk in diabetic and nondiabetic patients. J Clin Endocrinol Metab 2005;90:5698-703.

7 Bonora E, Kiechl S, Willeit J, et al. Insulin resistance as estimated by homeostasis model assessment predicts incident symptomatic cardiovascular disease in Caucasian subjects from the general population: the Bruneck study. Diabetes Care 2007;30:318-24

8 Schmiegelow MD, Hedlin H, Stefanick ML, et al. Insulin resistance and risk of cardiovascular disease in postmenopausal women: a cohort study from the women's health Initiative. Circ Cardiovasc Qual Outcomes 2015;8:309-16.

9 Rundek T, Gardener H, Xu Q, et al. Insulin resistance and risk of ischemic stroke among nondiabetic individuals from the Northern Manhattan study. Arch Neurol 2010;67:1195-200.

10 Thacker EL, Psaty BM, McKnight B, et al. Fasting and post-glucose load measures of insulin resistance and risk of ischemic stroke in older adults. Stroke 2011;42:3347-51.

11 Wieberdink RG, Koudstaal PJ, Hofman A, et al. Insulin resistance and the risk of stroke and stroke subtypes in the nondiabetic elderly. Am J Epidemiol 2012;176:699-707.

12 Lawlor DA, Harbord RM, Sterne JAC, et al. Mendelian randomization: using genes as instruments for making causal inferences in epidemiology. Stat Med 2008;27:1133-63.

13 Dormandy JA, Charbonnel B, Eckland DJA, et al. Secondary prevention of macroVascular events in patients with type 2 diabetes in the proactive study (prospective pioglitAzone clinical trial in macroVascular events): a randomised controlled trial. Lancet 2005;366:1279-89.

14 Kernan WN, Viscoli CM, Furie KL, et al. Pioglitazone after ischemic stroke or transient ischemic attack. N Engl J Med 2016;374:1321-31.
15 Liu J, Rutten-Jacobs L, Liu M, et al. Causal impact of type 2 diabetes mellitus on cerebral small vessel disease: a Mendelian randomization analysis. Stroke 2018;49:1325-31.

16 Marini S, Merino J, Montgomery BE, et al. Mendelian randomization study of obesity and cerebrovascular disease. Ann Neurol 2020;87:516-24.

17 Ross S, Gerstein HC, Eikelboom J, et al. Mendelian randomization analysis supports the causal role of dysglycaemia and diabetes in the risk of coronary artery disease. Eur Heart J 2015;36:1454-62.

18 Yaghootkar H, Scott RA, White CC, et al. Genetic evidence for a normal-weight "metabolically obese" phenotype linking insulin resistance, hypertension, coronary artery disease, and type 2 diabetes. Diabetes 2014;63:4369-77.

19 Tikkanen E, Pirinen M, Sarin A-P, et al. Genetic support for the causal role of insulin in coronary heart disease. Diabetologia 2016;59:2369-77.

20 Scott RA, Lagou V, Welch RP, et al. Large-Scale association analyses identify new loci influencing glycemic traits and provide insight into the underlying biological pathways. Nat Genet 2012;44:991-1005

21 Manning AK, Hivert M-F, Scott RA, et al. A genome-wide approach accounting for body mass index identifies genetic variants influencing fasting glycemic traits and insulin resistance. Nat Genet 2012;44:659-69.

22 Willer CJ, Schmidt EM, Sengupta S, et al. Discovery and refinement of loci associated with lipid levels. Nat Genet 2013;45:1274-83.

23 Nikpay M, Goel A, Won H-H, et al. A comprehensive 1,000 Genomes-based genome-wide association meta-analysis of coronary artery disease. Nat Genet 2015;47:1121-30.

24 Malik R, Chauhan G, Traylor M, et al. Multiancestry genome-wide association study of 520,000 subjects identifies 32 loci associated with stroke and stroke subtypes. Nat Genet 2018;50:524-37.

25 Burgess S, Butterworth A, Thompson SG. Mendelian randomization analysis with multiple genetic variants using summarized data. Genet Epidemiol 2013;37:658-65.

26 Bowden J, Davey Smith G, Burgess S. Mendelian randomization with invalid instruments: effect estimation and bias detection through Egger regression. Int J Epidemiol 2015;44:512-25.

27 Bowden J, Del Greco M F, Minelli C, et al. Assessing the suitability of summary data for two-sample Mendelian randomization analyses using MR-Egger regression: the role of the 12 statistic. Int $J$ Epidemiol 2016;45:dyw220-1974

28 Bowden J, Davey Smith G, Haycock PC, et al. Consistent estimation in Mendelian randomization with some invalid instruments using a weighted median estimator. Genet Epidemiol 2016;40:304-14.

29 Locke AE, Kahali B, Berndt SI, et al. Genetic studies of body mass index yield new insights for obesity biology. Nature 2015;518:197-206.

30 Knowles JW, Xie W, Zhang Z, et al. Identification and validation of $\mathrm{N}$-acetyltransferase 2 as an insulin sensitivity gene. J Clin Invest 2015;125:1739-51

31 Prins BP, Abbasi A, Wong A, et al. Investigating the causa relationship of C-reactive protein with 32 complex somatic and psychiatric outcomes: a large-scale Cross-Consortium Mendelian randomization study. PLoS Med 2016;13:e1001976.

32 Howard G, Wagenknecht LE, Kernan WN, et al. Racial differences in the association of insulin resistance with stroke risk: the reasons for geographic and racial differences in stroke (regards) study. Stroke 2014;45:2257-62.

33 Park H-Y, Park D-S, et al. Correlation between insulin resistance and intracranial atherosclerosis in patients with ischemic stroke without diabetes. J Stroke Cerebrovasc Dis 2008;17:401-5.

34 Sourij H, Schmoelzer I, Dittrich P, et al. Insulin resistance as a risk factor for carotid atherosclerosis: a comparison of the homeostasis model assessment and the short insulin tolerance test. Stroke 2008;39:1349-51.

35 Lee JE, Shin DW, Yun JM, et al. Insulin resistance is a risk factor for silent lacunar infarction. Stroke 2016;47:2938-44.

36 Allman PH, Aban IB, Tiwari HK, et al. An introduction to Mendelian randomization with applications in neurology. Mult Scler Relat Disord 2018;24:72-8.

37 Gan W, Walters RG, Holmes MV, et al. Evaluation of type 2 diabetes genetic risk variants in Chinese adults: findings from 93,000 individuals from the China Kadoorie Biobank. Diabetologia 2016;59:1446-57.

38 Gupta V, Walia GK, Sachdeva MP. 'Mendelian randomization': an approach for exploring causal relations in epidemiology. Public Health 2017:145:113-9. 